\title{
Clinicopathologic features and surgical management of primary umbilical melanoma: a case series
}

\author{
Gianluca Di Monta ${ }^{1 *}$, Corrado Caracò ${ }^{1}$, Ugo Marone ${ }^{1}$, Antonio Maria Grimaldi², Anna Maria Anniciello ${ }^{3}$, \\ Massimiliano Di Marzo ${ }^{1}$, Ester Simeone ${ }^{2}$ and Stefano Mori ${ }^{1}$
}

\begin{abstract}
Background: Primary umbilical melanoma is an uncommon tumor that is poorly described in the medical literature. The umbilical region is a particular anatomic site owing to the presence of embryonal remnants, which can be a potential metastatic pathway, as well as the braided lymphatic network drainage. Hence, primary malignant neoplasms affecting the umbilicus require a different and more radical surgical approach compared with other melanomas.

Case presentation: In this report, we describe a series of three patients of Caucasian ethnicity who presented with primary umbilical melanoma at the National Cancer Institute of Naples, Italy. All patients underwent wide excision of the tumor including the underlying peritoneum. No surgical complications, either immediate or delayed, were observed in any of the patients. Sentinel lymph node biopsy was negative in two cases. Two of the patients developed metastatic disease and died after systemic medical therapy. The other patient is currently in follow-up, and remains disease-free after 21 months.

Conclusions: The umbilicus has vascular and embryological connections with the underlying peritoneum, so that early visceral involvement is more likely to occur with primary umbilical melanomas. As such, tumor resection including the underlying peritoneum is required to avoid local relapse, whilst sentinel lymph node biopsy appears to be of poor diagnostic value.
\end{abstract}

Keywords: Primary umbilical melanoma, Umbilical tumor, Sentinel lymph node biopsy

\section{Background}

Primary umbilical melanoma is an uncommon disease, with only 23 cases described in the medical literature since it was first reported in 1916 [1] (Table 1). The umbilical region represents an anatomic site with unique characteristics, owing to the presence of embryonal remnants which provide a potential metastatic pathway, as well as the braided lymphatic drainage network. Hence, primary malignant neoplasms that affect the umbilicus require a different surgical approach compared with other melanomas [2]. In this report, we describe three patients who presented with primary umbilical melanoma at the National

\footnotetext{
* Correspondence: gidimonta@libero.it

'Department of Surgery "Melanoma, Soft Tissues, Head and Neck, Skin Cancers", National Cancer Institute "G. Pascale", Via M. Semmola, 80131 Naples, Italy

Full list of author information is available at the end of the article
}

Cancer Institute of Naples, Naples, Italy, between May 2010 and April 2011.

\section{Case presentation \\ Case 1}

A 33-year-old Caucasian female presented with a coarse nodular pinkish-brown swelling that covered the natural umbilical cavity. Diagnosis of a nodular melanoma was based on clinical findings, with histological investigation showing a Breslow thickness of $40 \mathrm{~mm}$, ulceration, mitotic rate of $6 / \mathrm{mm}^{2}$ and no tumor-infiltrating lymphocytes (Figure 1). Whole-body fluorodeoxyglucose positron emission tomography/computed tomography (FDG PET/CT) was negative for distant disease. The patient underwent pre-operative $\mathrm{Tc}^{99}$ lymphoscintigraphy according to standard procedure and subsequent removal of the umbilical 
Table 1 Review of literature

\begin{tabular}{|c|c|c|c|}
\hline Author & Year & $\begin{array}{l}\text { Number } \\
\text { of patients }\end{array}$ & Treatment \\
\hline Cullen TS [5] & 1916 & 3 & Excision \\
\hline Graev M [6] & 1957 & 1 & Excision \\
\hline Hughes J [7] & 1963 & 1 & Excision \\
\hline Steck WD [8] & 1965 & 4 & Excision \\
\hline Breuninger H [9] & 1996 & 7 & $\begin{array}{l}\text { Excision and plastic } \\
\text { reconstruction }\end{array}$ \\
\hline Colonna MR [10] & 1999 & 2 & $\begin{array}{l}\text { Excision and plastic } \\
\text { reconstruction }\end{array}$ \\
\hline Meine JG [2] & 2003 & 1 & $\begin{array}{l}\text { Excision and plastic } \\
\text { reconstruction }\end{array}$ \\
\hline Campos-Muñoz [11] & 2007 & 1 & $\begin{array}{l}\text { Excision, SLNB and } \\
\text { plastic reconstruction }\end{array}$ \\
\hline Mangas C [12] & 2008 & 1 & $\begin{array}{l}\text { Excision and plastic } \\
\text { reconstruction }\end{array}$ \\
\hline Cecchi R [3] & 2009 & 1 & Excision and SLNB \\
\hline Zaccagna A [1] & 2011 & 1 & $\begin{array}{l}\text { Excision, SLNB and } \\
\text { plastic reconstruction }\end{array}$ \\
\hline Current study & 2014 & 3 & $\begin{array}{l}\text { Excision, SLNB and } \\
\text { plastic reconstruction }\end{array}$ \\
\hline Total & & 26 & \\
\hline
\end{tabular}

region, which consisted of the tumor with $2 \mathrm{~cm}$ margins including the underlying peritoneum. Sentinel lymph node biopsy (SLNB) in the left inguinal and bilateral axillary regions, as indicated by lymphoscintigraphy, were negative for metastases. Six months after surgery, there was local recurrence involving the posterior aspect of the left rectus muscle up to the peritoneum. The patient underwent surgical removal of both rectus muscles including the local recurrence (Figure 2), with the abdominal wall being reconstructed with non-absorbable Marlex mesh.

However, the patient subsequently developed multiple inoperable nodal and abdominal metastases. A BRAF mutation test was positive and, because BRAF mutation is associated with enhanced and selective sensitivity to MEK inhibition when compared to either wild-type cells or cells with a RAS mutation, the patient was enrolled in an experimental clinical protocol involving treatment with a selective MEK inhibitor (MEK162 $15 \mathrm{mg}$ orally). After four months, an ${ }^{18}$ FDG PET/CT scan showed a significant response to treatment with minor intestinal ${ }^{18} \mathrm{FDG}$ accumulation in peritoneal thickening close to the bladder (maximum standardized uptake value $\left[\mathrm{SUV}_{\max }\right]$ of 3.6), and the disappearance of lymphadenopathy and swellings in the subcutaneous fat pad of the abdominal wall. Therapy was continued for three months, until disease progression was observed on a total body CT scan.

Considering the BRAF mutation-positive status of the patient, treatment was switched to oral vemurafenib $240 \mathrm{mg}$, a selective BRAF inhibitor. However, this was stopped after 8 weeks due to clinical disease progression. The patient then began a new line of therapy with ipilimumab $3 \mathrm{mg} / \mathrm{kg}$ as part of an Italian expanded access programme. Six months later the patient received her fourth administration of ipilimumab, and was completely free of ascites and/or bowel symptoms. The patient died 28 months after initial diagnosis of the disease.

\section{Case 2}

A 50-year-old Caucasian female had previously undergone removal of a pigmented lesion of the umbilical region at a hospital other than our institute, with histological findings of superficial spreading melanoma, Breslow thickness $2.5 \mathrm{~mm}$, ulceration, mitotic rate of $6 / \mathrm{mm}^{2}$ and brisk tumor-infiltrating lymphocytes. After referral to our centre, the umbilical region, including the scar from the initial excision of the lesion, with a $2 \mathrm{~cm}$ lateral margin including the underlying peritoneum was surgically removed (Figure 3). The reconstruction of the umbilicus was carried out by means of two local transposition flaps transferred towards the linea alba. SLNB in the right inguinal
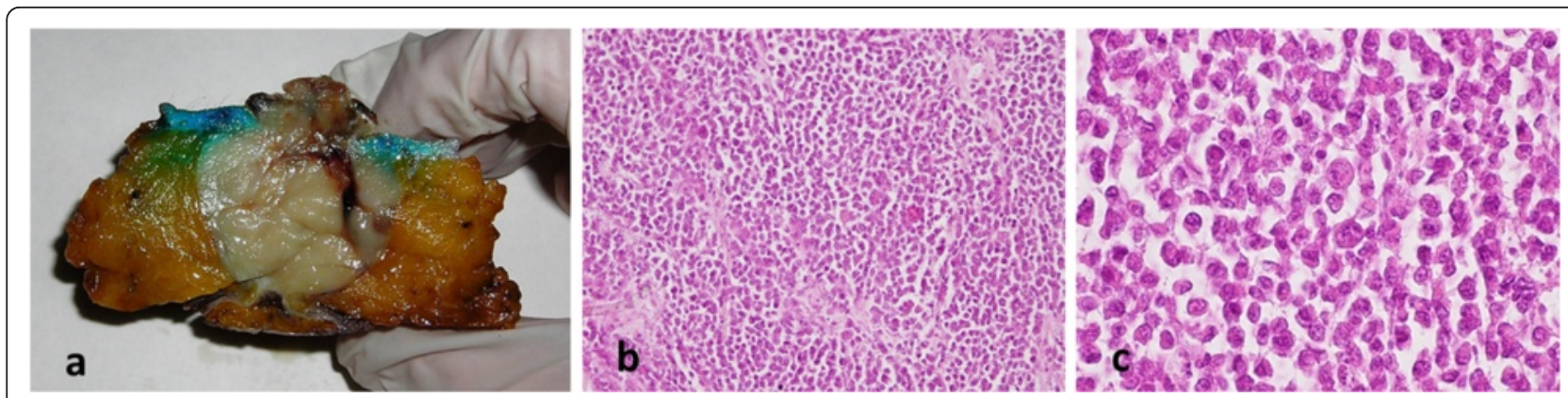

Figure 1 Case 1. (a) Specimen cut in half: Umbilicus cavity is completely filled by tumor (b) poorly differentiated cells, that look primitive, disorganized and immature (hematoxylin and eosin, $\times 10$ ) (c) diffused anisocytosis, anisonucleosis, altered nuclear-cytoplasmic ratio and evident nucleoli (hematoxylin and eosin, $\times 60$ ). 


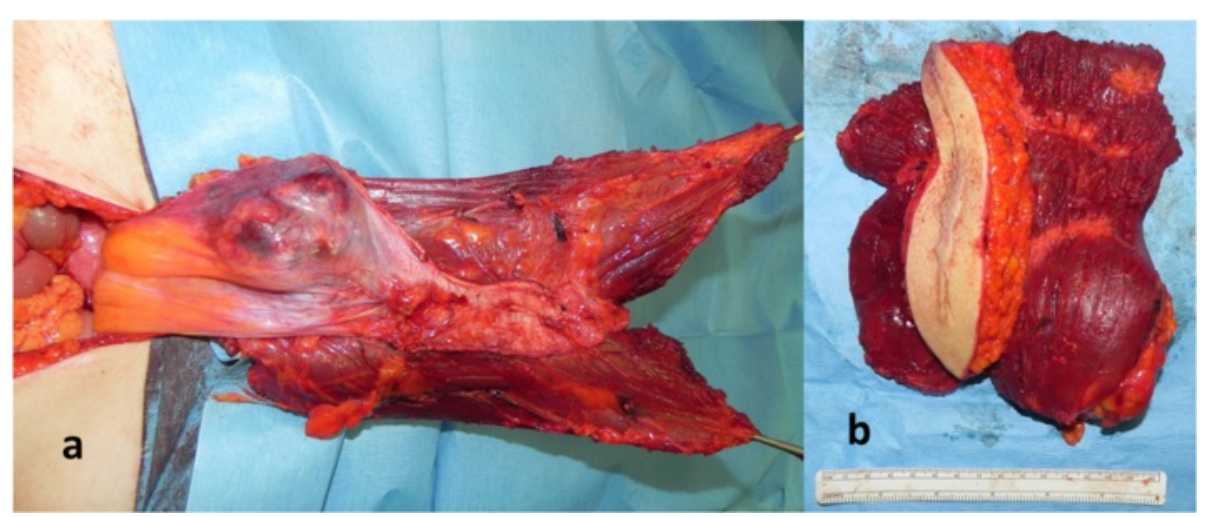

Figure 2 Case 1 local recurrence. (a) Local recurrence involving the left rectus muscle up to the peritoneum (b) removal of both rectus muscles including the overlying skin bearing the scar of previous excision.

basin was negative for metastases. The patient remains disease-free after 21 months of follow-up.

\section{Case 3}

A 77-year-old Caucasian male had previously undergone removal of a pigmented lesion in the umbilical region at another hospital. Histology showed an ulcerated cutaneous melanoma of $3 \mathrm{~mm}$ thickness and mitotic rate of $6 / \mathrm{mm}^{2}$. No further treatment was administered until, twelve months later, the patient presented with a swelling underlying his umbilicus. Clinical and instrumental examinations showed a coarse nodular swelling in the umbilical region, most likely local disease recurrence, and major lymphadenopathy of $54 \times 28 \mathrm{~mm}$ in the left groin, which was positive in pre-operative cytology. The patient underwent left superficial and deep groin dissection and omphalectomy, with $2 \mathrm{~cm}$ of lateral margin extended up to the underlying peritoneum (Figure 4). The patient then underwent medical treatment of subsequent bone and liver metastases but died eight months later due to disseminated disease.

\section{Discussion}

Melanoma of the umbilical region is a rare and particularly aggressive neoplastic disease. Primary umbilical malignancies are very poorly described, consisting of melanomas, basal cell and squamous cell carcinomas. The umbilicus has vascular and embryological connections with the underlying peritoneum, with the urachus, vitelline artery and round ligament of the liver representing the main metastatic spread pathway. Primary umbilical melanoma determines a possible early visceral involvement and so should be approached with a wide excision of the entire umbilical structure, including the attachment to the underlying peritoneum [3].

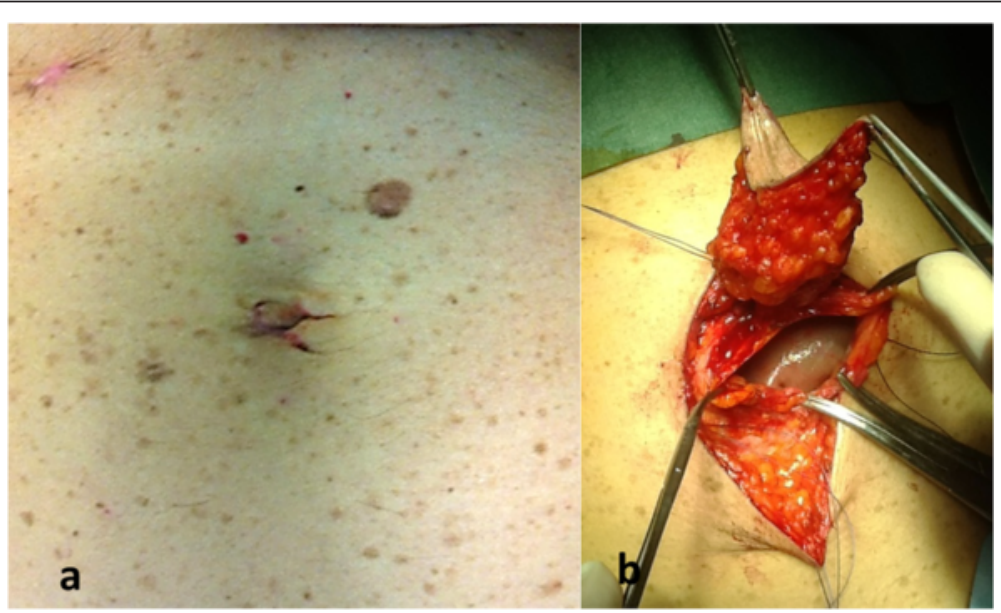

Figure 3 Case 2. (a) Pre-operative view (b) wide excision comprising the underlying peritoneum. 


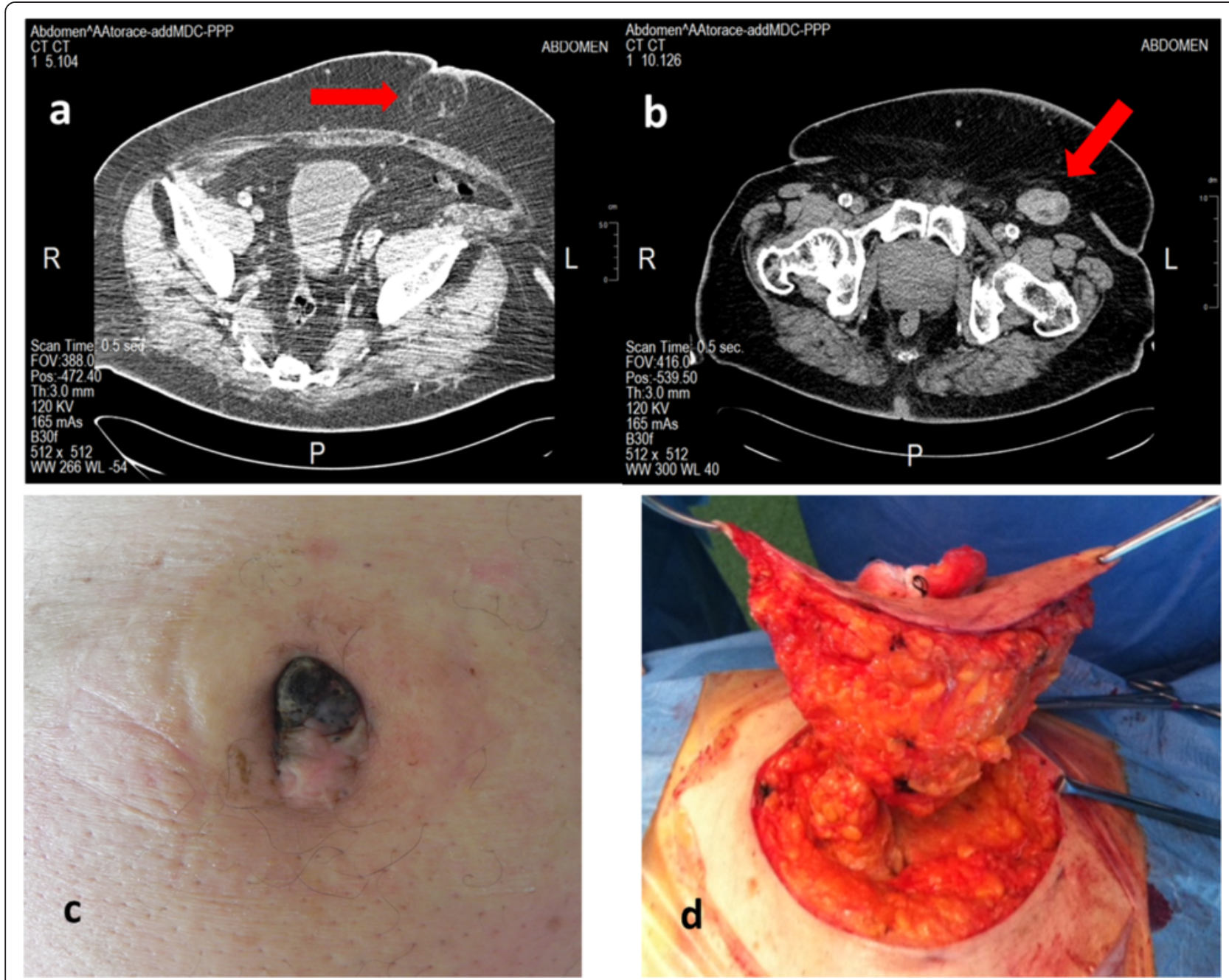

Figure 4 Case 3. (a) Computed tomography image showing nodular swelling in the umbilical region (b) Computed tomography image of left groin lymphadenopathy of $54 \times 28$ mm (c) pre-operative view (d) wide excision comprising the underlying peritoneum.

In the three patients reported here, the first died 12 months after the second surgery. The second patient is currently in follow-up, and remains disease-free after 21 months. In the third patient, bone and liver metastases developed a year after the diagnosis of primary umbilical melanoma and led to the patient's death. No surgical complications, either immediate or delayed, were observed in any of the patients. The medical oncology staff treated two of the three patients for systemic disease.

The difference between primary umbilical melanoma and metastatic tumors of the umbilicus should be noted. Metastatic tumors account for approximately $80-88 \%$ of malignancies occurring in the umbilical area $[3,4]$. The classic example is the "Sister Mary Joseph nodule," representing a metastasis from the gastrointestinal tract. In a review of 77 umbilical malignancies at a single centre, the most common primary sites with metastasis to the umbilicus were the ovary, endometrium, and pancreatobiliary tree in women, and the genitourinary tract, pancreatobiliary tree, and gastrointestinal tract in men [2-4].

SLNB for primary umbilical melanomas are very rarely reported. The umbilicus has a lymphatic drainage involving axilla and inguinal areas or, more rarely, internal mammary, iliac or intra-abdominal lymph nodes [1]. In our patients, lymphoscintigraphy showed the presence of the sentinel lymph node in both armpits and left groin in the first patient and in the right groin in the second patient. As such, the potential role of SLNB of umbilical tumors represents an unanswered question but at present has to be considered of poor diagnostic value.

\section{Conclusions}

Primary umbilical melanoma should be considered a different pathology from common cutaneous melanoma. Tumor 
excision en bloc with the underlying peritoneum is required to avoid local relapse. Immediate reconstruction of the neo-umbilicus with local flaps should be carried out at the same time, while respecting the aggressive nature of the neoplasm. SLNB appears to be of poor diagnostic value.

\section{Consent}

Written informed consent was obtained from the next of kin of both dead patients and from the patient still alive for publication of this Case Report and accompanying images. A copy of the written consent is available for review by the Editor-in-Chief of this journal.

\section{Abbreviations}

FDG PET/CT: Fluorodeoxyglucose positron emission tomography/computed tomography; SLNB: Sentinel lymph node biopsy; SUV max: Maximum standardized uptake value.

\section{Competing interests}

The authors declare that they have no competing interest. There is no external source of funding involved in the submitted article.

\section{Authors' contributions}

GDM and CC conceived the study and drafted the manuscript. UM and MDM helped in the preparation of the manuscript and carried out the literature research. AMG and ES performed medical oncology therapies and helped in management of the patients. AMA performed pathologic study. SM and CC realized the technique. All authors read and approved the final manuscript.

\section{Author details}

${ }^{1}$ Department of Surgery "Melanoma, Soft Tissues, Head and Neck, Skin Cancers", National Cancer Institute "G. Pascale", Via M. Semmola, 80131 Naples, Italy. ${ }^{2}$ Unit of Melanoma, Cancer Immunotherapy and Innovative Therapy, National Cancer Institute "G. Pascale", 80131 Naples, Italy. ${ }^{3}$ Department of Pathology, National Cancer Institute "G. Pascale", 80131 Naples, Italy.

Received: 7 January 2014 Accepted: 25 March 2015

Published online: 15 April 2015

\section{References}

1. Zaccagna A, Siatis D, Pisacane A, Giacone E, Picciotto F. Surgical treatment of primary melanoma of the umbilicus with sentinel lymph node biopsy and plastic reconstruction: case report and review of the literature. Eur J Surg Oncol. 2011;37:233-6.

2. Meine JG, Bailin PL. Primary melanoma of the umbilicus: report of a case and review of the relevant anatomy. Dermatol Surg. 2003;29:405-7.

3. Cecchi R, Pavesi M, Buralli L, Rapicano V, De Gaudio C. Primary umbilical melanoma. Australas J Dermatol. 2009:50:220-2.

4. Papalas JA, Selim MA. Metastatic vs primary malignant neoplasms affecting the umbilicus: clinicopathologic features of 77 tumors. Ann Diagn Pathol. 2011;15:237-42.

5. Cullen TS. Embryology, anatomy, and diseases of the umbilicus, together with disease of the urachus. Philadelphia: WB Saunders; 1916. p. 457-8.

6. Graev M. First known case of primary malignant melanoma of the umbilicus. Arch De Vecchi Anat Patol. 1957;27:589-602.

7. Hughes J. Melanoma of the umbilicus: a case report. J Ir Med Assoc. 1963:53:94-8.

8. Steck WD, Helwig EB. Tumors of the umbilicus. Cancer. 1965;18:907-15.

9. Breuninger $\mathrm{H}$, Zimmermann $\mathrm{C}$. Umbilical reconstruction after excision of melanomas in the area of the umbilicus. Hautartz. 1996;47:273-5.
10. Colonna MR, Giovannini UM, Sturniolo G, Colonna U. The umbilicus: a rare site for melanoma. clinical considerations in two cases. Scand J Plast Reconstr Hand Surg. 1999;33:449-52.

11. Campos-Muñoz L, Quesada-Cortés A, Ruiz E, Casado M, Pizarro A. Primary melanoma of the umbilicus appearing as omphalitis. Clin Exp Dermatol. 2007;32:322-4.

12. Mangas C, Romani J, Muñoz C, Luelmo J. Navel melanoma: not always easy to detect, not always difficult to remove. Dermatol Online J. 2008;14:20. 15.

\section{Submit your next manuscript to BioMed Central and take full advantage of:}

- Convenient online submission

- Thorough peer review

- No space constraints or color figure charges

- Immediate publication on acceptance

- Inclusion in PubMed, CAS, Scopus and Google Scholar

- Research which is freely available for redistribution 